\title{
Nonintrusive proper generalized decomposition method for the design optimization of a car
}

\author{
F. Cavaliere ${ }^{1,2}$, S. Zlotnik ${ }^{1}$, R. Sevilla ${ }^{2}$, X. Larráyoz ${ }^{3}$ and P. Díez ${ }^{1}$ \\ ${ }^{1}$ Laboratori de Càlcul Numèric (LaCàN), Campus Norte UPC, 08034 Barcelona, Spain \\ e-mail: fabiola.cavaliere1@upc.edu, sergio.zlotnik@upc.edu, pedro.diez@upc.edu \\ 2 Zienkiewicz Centre for Computational Engineering, Swansea University, Wales, UK \\ e-mail: r.sevilla@swansea.ac.uk \\ ${ }^{3}$ Centro Técnico de SEAT S.A., Autovía A-2, km 85, Martorell 08760, Spain \\ e-mail: xabier.larrayoz@seat.esg
}

\begin{abstract}
A lot of estabilished target specifications have to be respected during the pre-design process of the car body-in-white (BIW) stucture. One important target is represented by the noise and vibration characteristics of the vehicle (NVH test), which strictly depends on the global static and dynamic stiffness. These characteristics might be significantly affected by changes in the material and geometric properties of the car components. In order to explore all the possible combinations for the whole range of the parameters involved, an extremely high number of configurations should be tested. Due to the high computational cost of each simulation, classical numerical methods become extremely time consuming when applied to parametric studies as the one under discussion. Several reduced order method (ROM) techniques were developed in the last decades, all with the common goal of finding low-order models, described by a reduced order basis, which are able to capture the essential behaviour of a complex system. In this work, the encapsulated proper generalized decomposition (Encapsulated-PGD) toolbox, based on the PGD Least-Squares approximation [1] is proposed. This tool is able to provide, with only one offline computation, an explicit separable solution in terms of an a-priori unknown number of parametric and mechanic modes or snapshots. Then, during an online stage, the solution can be particularized for any set of the parameters in realtime. Here, the method has been tested with respect to different problems of interest [2]. First, the PGD method was coupled with the Inertia Relief technique [3] in order to perform the parametric static analysis of unconstrained structures. Then, the Encapsulated-PGD toolbox was used to solve a parametric generalized eigenvalue problem in order to identify how a variation of userdefined parameters affects the dynamic response of the structure in terms of dominant eigenmodes and related natural frequencies. Thanks to a novel algebraic approach, the method allows to deal with material and complex geometric parameters. Moreover, the nonintrusive format of the toolbox allows the interaction with commercial software, making it available for real industrial applications.
\end{abstract}

\section{REFERENCES}

[1] Díez, Pedro and Zlotnik, Sergio and García-González, Alberto and Huerta, Antonio. Encapsulated PGD Algebraic Toolbox Operating with High-Dimensional Data, Arch Computat Methods Eng, doi:10.1007/s11831-019-09378-0, (2019).

[2] Cavaliere, F and Zlotnik, S and Sevilla, R and Larrayoz, X and Diez, P, Nonintrusive reduced order model for parametric solutions of inertia relief problems, arXiv preprint arXiv:2012.01961, (2020).

[3] Barnett, Alan R and Widrick, Timothy W and Ludwiczak, Damian R Closed-form static analysis with inertia relief and displacement-dependent loads using a MSC/NASTRAN DMAP Alter, (1995). 